\title{
Short-term Outcomes of a Laparoscopic Left Hemicolectomy for Descending Colon Cancer: Retrospective Comparison with an Open Left Hemicolectomy
}

\author{
Kil-Su Han, Gyu-Seog Choi, Jun-Seok Park, Hye Jin Kim, Soo Yeon Park, Soo-Han Jun \\ Department of Surgery, Kyungpook National University Hospital, Kyungpook National University School of Medicine, Daegu, Korea
}

Purpose: Many randomized clinical trials have been performed to treat a colorectal neoplasm with the exclusion of descending colon cancer. The aim of the present study was to investigate the difference in surgical outcomes between a laparoscopic left hemicolectomy and a conventional open left hemicolectomy for descending colon cancer.

Methods: A retrospective study of ninety patients with descending colon cancer, who underwent a laparoscopic (LAP) or open left hemicolectomy (OS) between May 1998 and December 2009 at Kyungpook National University Hospital, was performed. Clinicopathological and surgical outcomes were compared between the LAP and the OS for descending colon cancer.

Results: The baseline characteristics, including age, gender, body mass index, history of prior abdominal surgical history and tumor location, were similar between the two groups. The mean operation time was 156.2 minutes for the LAP group and 223.2 minutes for the OS group $(\mathrm{P}<0.001)$. Intraoperative blood loss was significantly greater in the OS group $(37.5$ $\mathrm{mL}$ vs. $80.4 \mathrm{~mL} ; \mathrm{P}=0.039$ ). The postoperative recovery in the LAP group was faster, as reflected by the shorter time to pass gas and the shorter hospital stay. Pathological examinations showed the surgery to be equally radical in the two groups. The median follow-up was 21 months and there were 3 distant metastases (8.5\%) during follow-up in the LAP group, but no port-site or local recurrence.

Conclusion: A laparoscopic left hemicolectomy is a technically safe and feasible procedure for treating descending colon cancer. Prospective multi-center trials are necessary to establish the LAP as the standard treatment for descending colon cancer.

Keywords: Descending colon cancer; Left hemicolectomy; Laparoscopy; Surgical outcomes

\section{INTRODUCTION}

In regard to laparoscopic surgical treatments for colon cancer, safety and complete cure have already been assessed by several random comparison studies, and the results show advantages

Received: August 31, 2010 Accepted: September 16, 2010 Correspondence to: Gyu-Seog Choi, M.D.

Department of Surgery, Kyungpook National University School of Medicine, 50 Samduck-dong 2ga, Jung-gu, Daegu 700-721, Korea

Tel: +82-53-420-5619, Fax: +82-53-421-0510

E-mail: kyuschoi@knu.ac.kr

(C) 2010 The Korean Society of Coloproctology

This is an open-access article distributed under the terms of the Creative Commons Attribution NonCommercial License (http://creativecommons.org/licenses/by-nc/3.0) which permits unrestricted noncommercial use, distribution, and reproduction in any medium, provided the original work is properly cited such as less manipulation of the intestine, minimized postsurgical pain and shortened hospital stay; thus, the return to routine life was fast [1-5]. Therefore, indications for laparoscopic surgery for the treatment of colon cancer have been expanding gradually.

Cancer in the descending colon, including the splenic flexure, accounts for 5-6\% of all colon cancer; thus, its incidence is low. Colon cancer easily causes obstruction so emergency treatment is required in many cases [6-8]. In addition, descending colon cancer has the lymphoid system draining to the superior mesenteric artery and the inferior mesenteric artery, so problems are that the lack of standardized lymph node dissection and technical difficulties pertinent to splenic flexure dissection essential to providing sufficient resection margins. Therefore, in prospective multicentric randomized studies, together with 
transverse colon cancer patients, descending colon cancer patients were frequently excluded as study subjects. Although the number of patients was limited, in this study, the authors investigated the effectiveness of laparoscopic surgery and tentative indications by comparing clinical outcomes for laparoscopy-assisted left hemicolectomies (LAP) and to those for conventional open left hemicolectomies (OS) performed around the same period.

\section{METHODS}

\section{Subjects}

The study was conducted on patients who underwent surgical treatment for primary cancer in the splenic flexure or the descending colon from May 1998 to December 2009 at our hospital. Among them, patients who received a transverse colon segmental resection, an extended right hemicolectomy, or a subtotal colectomy, patients with synchronous primary cancers, patients on whom a co-resection of other major organs had been performed, patients with cancer in the sigmoid-descending colon junction, and patients on whom emergency surgery for obstruction or perforation had been performed were excluded from the analysis.

As presurgical diagnostic tests, serum carcino-embryonic antigen (CEA) test, chest radiography, colonoscopy, abdominal computed tomography, and positron emission transmission computed tomography (PET-CT) were performed. Descending colon cancer was defined as tumors located in the area between the proximal splenic flexure and the sigmoid colon-descending colon junction detected by presurgical tests such as computed tomography, colonoscopy, or PET-CT, and all lesions were adenocarcinomas, as confirmed by histological tests (Fig. 1). Disease stages were classified according to the American Joint Committee on Cancer (AJCC) tumour node metastasis (TNM) classification, 6th edition. This study was a retrospective study, and no special standards were applied to select the patients for laparoscopic or open abdominal surgery for descending colon cancer. The authors performed a laparoscopic left hemicolectomy in February 2002 for the first time. Initially, a laparoscopic left hemicolectomy was limited to patients whose lesion was less than T3 by abdominal computed tomography and who had no past history of major abdominal surgery. As the surgeons have acquired experiences and have become skilled, presently, at our hospital, the principle is to perform laparoscopic surgery except in cases with wide spread invasion to adjacent organs, obstruction of the intestine associated with abdominal distention, and tumors larger than $8 \mathrm{~cm}$. We compared and analyzed retrospectively the characteristics of patients that are eliciated from prospectively collected database of our hospital and medical records, such as the ages of the patients, the gender ratio, body mass index $\left(\mathrm{BMI}, \mathrm{kg} / \mathrm{m}^{2}\right)$, surgical methods, cases converted to laparotomy, operation time, hemorrhage volume, time of the first passing gas, time of the first oral intake, postsurgical hospitalization period, complications, and oncological results (distance from tumors to resection margins, the numbers of resected lymph nodes, and recurrence patterns).

\section{Surgical methods}

In our study, a radical left hemicolectomy was defined as the complete removal of the primary tumor and lymph node resection, including the origin of the left colic artery. The laparoscopic surgery and laparotomy were performed by the same two surgeons or under their supervision.

\section{Laparoscopic surgery}

All surgeries were performed under general anesthesia, and abdominal pressure was maintained so as not to exceed 12 $\mathrm{mmHg}$. In all patients, the presence of metastasis in the entire abdominal cavity, including the liver, was assessed directly macroscopically after inserting a laparoscope. In the modified lithotomy position, a 11-mm trocar was installed below the umbilicus, and a 0 -degree camera was inserted. Five-mm trocars were inserted at the right upper abdomen and the right lower abdomen and were used by the operator. A 5-mm trocar was inserted at the left side and was used by the first assistant. On the right side of the patient, the operator and a camera assistant were positioned, and on the left side, the first assistant was positioned. Surgery was initiated by resecting soft tissues, including lymph nodes, while paying attention not to injure the sympathetic nerves around the inferior mesenteric artery. The origin of the left colic artery branching from the inferior mesenteric artery was assessed and ligated with clips.

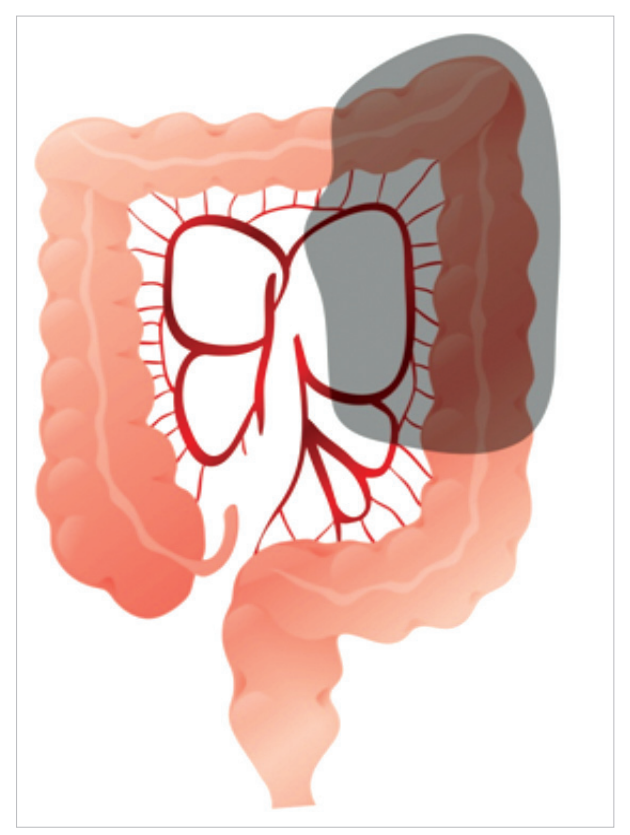

Fig. 1. The extent of the left hemicolectomy. 
While assessing the location of the ureter and gonadal vessels, the descending mesocolon was resected from the retroperitoneum from the medial to the lateral direction. Regarding the lateral side, resection was performed beyond the Gerota's fascia to the left abdominal wall. Concerning the cephalad direction, resection was performed until the pancreas was detected. At that time, the origin of the inferior mesenteric vein was assessed and ligated, lymph node dissection in the mid colic artery and its left branch area was performed and ligated. After sufficient resection of the medial side, the descending colon was pulled to the medial side, and the Toldt's fascia was dissected in order to separate the descending colon from the lateral wall from the iliac ridge to the splenic flexure. When the proximal sigmoid colon and the descending colon had been completely separated from the retroperitoneum, while resecting the greater omentum of the transverse colon, the right gastrocolic ligament was separated. After complete mobilization of the splenic flexure area had been confirmed, the umbilical port was extended and a mini laparotomy $4-6 \mathrm{~cm}$ in size was made. After the installation of wound protectors, the descending and the sigmoid colon were extracted, and the distal transverse colon and the proximal sigmoid colon were side-to-end anastomosed by using a circular end-to-end anastomosis stapler.

\section{Open surgery}

The range and the method of surgery were almost identical to those for a LAP; nonetheless, as reconstruction, a manual endto-end anastomosis was performed on the distal transverse colon and the proximal sigmoid colon. The range of lymph node resection and the range of omentectomy were not different in principle from those for laparoscopic surgery.

\section{Analysis of the results and statistical analysis}

For analyzing the characteristic of the patients and the clinical outcomes, the means and standard deviations were examined. For the comparisons of the variables of the two surgery groups, the independent sample t-test and the cross-table Pearson chisquare test were applied. For statistical analysis, the SPSS ver. 15.0 (SPSS Inc., Chicago, IL, USA) was used. A P-value less than 0.05 was considered to be statistically significant.

\section{RESULTS}

From 1998 to December 2009, a total of 281 patients received a radical resection for distal transverse colon cancer, splenic flexure or descending colon cancer. Among them, 90 patients received a left hemicolectomy or extended left hemicolectomy. Among those 90, a laparoscopic left hemicolectomy was performed in 35 cases.

\section{Clinico-pathological characteristic}

The mean age of the subject patients of the LAP group was
58.6 years (range, 33 to 84 years), and that of the OS group was 59.1 years (range, 23 to 81 years). In regard to the gender of the LAP group, 28 patients were males, and 7 patients were females. In the OS group, 38 patients were males, and 17 patients were females. Regarding the other characteristics of the patient groups, the BMIs of the two groups, the presence or absence of associated diseases, and the past history of abdominal surgery prior to surgery were not significantly different. The locations of the tumors for the LAP group were the distal transverse colon in 3 patients $(8.6 \%)$, the splenic flexure in 16 patients (45.7\%), and the descending colon in 16 patients (45.7 $\%)$. In the OS group, the locations were the distal transverse colon in 5 patients $(5.5 \%)$, the splenic flexure in 12 patients $(21.8 \%)$, and the descending colon in 38 patients $(69.1 \%)(\mathrm{P}=$ 0.060). Presurgical serum CEA values for the LAP group and the OS group were $2.2 \pm 1.7 \mathrm{ng} / \mathrm{mL}$ and $5.3 \pm 7.8 \mathrm{ng} / \mathrm{mL}$, respectively, and this difference was significant $(\mathrm{P}=0.015)$ (Table 1$)$.

\section{Surgery results}

None of the patients on whom laparoscopic surgery was attempted were converted to a laparotomy. The mean operation time of the LAP was 156.2 minutes (range, 75 to 241 minutes), and that of the OS group was 223.2 minutes (range, 120 to 350 minutes), that of the LAP group being significantly shorter ( $\mathrm{P}$ $<0.001)$. The first time of passing gas after surgery for the LAP group was an average of 1.9 days (1-5 days) while that for the OS group was an average of 3.5 days (range, 2 to 8 days), being significantly shorter in the LAP group $(<0.001)$. The time to intake of normal meals after surgery for the LAP group was an average of 4.8 days (range, 3 to 11 days) while that for the

Table 1. Patient characteristics

\begin{tabular}{|c|c|c|c|}
\hline & $\operatorname{LAP}(n=35)$ & OS $(n=55)$ & P-value \\
\hline Age at surgery $(y r)^{a}$ & $58.6(11.6)$ & $59.1(14.0)$ & 0.845 \\
\hline $\begin{array}{l}\text { Sex }(\%) \\
\text { Male } \\
\text { Female }\end{array}$ & $\begin{array}{r}28(80.0) \\
7(20.0)\end{array}$ & $\begin{array}{l}38(69.1) \\
17(30.9)\end{array}$ & 0.254 \\
\hline BMI $\left(\mathrm{kg} / \mathrm{m}^{2}\right)^{\mathrm{a}}$ & $23.9(2.8)$ & $23.0(2.3)$ & 0.100 \\
\hline $\begin{array}{l}\text { ASA score }(\%) \\
\text { I } \\
\| \\
\text { III }\end{array}$ & $\begin{array}{c}10(28.6) \\
23(65.7) \\
2(5.7)\end{array}$ & $\begin{array}{c}29(52.7) \\
24(43.6) \\
2(3.6)\end{array}$ & 0.080 \\
\hline $\begin{array}{l}\text { Tumor location (\%) } \\
\text { Descending } \\
\text { Splenic flexure } \\
\text { Distal transverse colon }\end{array}$ & $\begin{array}{c}16(45.7) \\
16(45.7) \\
3(8.6)\end{array}$ & $\begin{array}{c}38(69.1) \\
12(21.8) \\
5(9.1)\end{array}$ & 0.060 \\
\hline Preoperative serum CEA (ng/mL) ${ }^{a}$ & $2.2(1.7)$ & $5.3(7.8)$ & 0.015 \\
\hline Prior abdominal surgery (\%) & $1(2.9)$ & $3(5.5)$ & 0.493 \\
\hline
\end{tabular}

LAP, laparoscopic surgery; OS, open left hemicolectomy; BMl, body mass index; ASA, American Society of Anesthesiologists; CEA, carcinoembryonic antigen.

aValues are presented as means (standard deviations); 'Included hepatic-flexure located tumor. 
Table 2. Intraoperative and postoperative outcomes

\begin{tabular}{|c|c|c|c|}
\hline & $\operatorname{LAP}(n=35)$ & OS $(n=55)$ & P-value \\
\hline $\begin{array}{l}\text { Extent of node dissection (\%) } \\
\text { IMA high ligation } \\
\text { IMA preservation }\end{array}$ & $\begin{array}{r}6(17.1) \\
29(82.9)\end{array}$ & $\begin{array}{l}11(20.0) \\
44(80.0)\end{array}$ & 0.736 \\
\hline Operation time $(\mathrm{min})^{\mathrm{a}}$ & $156.2(49.1)$ & $223.2(50.7)$ & $<0.001$ \\
\hline Estimated blood loss (mL) ${ }^{a}$ & $37.5(44.1)$ & $80.4(109.5)$ & 0.039 \\
\hline Conversion to open surgery (\%) & $0(0.0)$ & - & - \\
\hline Diverting stoma (\%) & $0(0.0)$ & $0(0.0)$ & 1.000 \\
\hline Flatus passage (day) $)^{a}$ & $1.9(0.7)$ & $3.5(1.4)$ & $<0.001$ \\
\hline Time to resume regular diet (day) $)^{a}$ & $4.8(1.9)$ & $6.1(1.5)$ & 0.016 \\
\hline Length of stay (day) ${ }^{\mathrm{a}}$ & $8.7(5.0)$ & $12.2(7.3)$ & 0.014 \\
\hline $\begin{array}{l}\text { Total morbidity }(\%)^{\mathrm{b}} \\
\text { Wound infection } \\
\text { Anemia requiring transfusion } \\
\text { Leakage } \\
\text { lleus } \\
\text { Enteritis } \\
\text { Incisional hernia } \\
\text { Atelectasis }\end{array}$ & $\begin{array}{l}5(14.3) \\
1(2.8) \\
2(5.7) \\
0(0.0) \\
1(2.8) \\
0(0.0) \\
1(2.8) \\
1(2.8)\end{array}$ & $\begin{array}{l}11(20.0) \\
4(7.2) \\
1(1.8) \\
1(1.8) \\
2(3.6) \\
1(1.8) \\
0(0.0) \\
2(3.6)\end{array}$ & 0.489 \\
\hline $\begin{array}{l}\text { Grade of morbidity (\%) } \\
\text { Dindo I-II } \\
\text { Dindo III-IV }\end{array}$ & $\begin{array}{c}34(97.2) \\
1(2.8)\end{array}$ & $\begin{array}{c}54(98.2) \\
1(1.8)\end{array}$ & 0.334 \\
\hline Mortality & $0(0.0)$ & $0(0.0)$ & - \\
\hline
\end{tabular}

LAP, laparoscopic surgery; OS, open left hemicolectomy; IMA, inferior mesenteric artery.

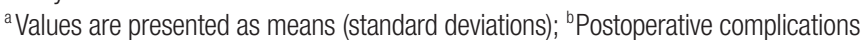
after 90-day follow-up.

OS group was an average of 6.1 days (range, 5 to 12 days), being significantly shorter in the LAP group $(\mathrm{P}=0.016)$. The hospitalization period after surgery for the LAP group was an average of 8.7 days (range, 6 to 55 days), and that of the OS group was an average of 12.2 days (range, 8 to 40 days), being significantly shorter in the LAP group $(\mathrm{P}=0.014)$. As postsurgical complications, in the LAP group, reoperation for herniation pertinent to the wound occurred in one case. In the OS group, reoperation for obstruction of the small intestine after surgery occurred in one case (Table 2).

\section{Morbidity and mortality}

In the LAP group, morbidity was $14.3 \%$ (5 patients out of the total 35 patients), and mortality was $0 \%$. In the OS group, morbidity was $20.0 \%$ (11 patients out of the total 58 patients), and mortality was $0 \%$ (Table 2 ). In regard to complications, in the LAP group, infection in the wound area occurred in 1 case, hemorrhage after surgery in 2 cases, obstruction of the intestine in 1 case, an incision hernia in 1 case, and lung infection due to atelectasis in 1 case. In the OS group, wound infection occurred in 4 cases, hemorrhage after surgery in 1 case, leakage in the anastomosis area in 1 case, obstruction of the intestine in 2 cases, colitis associated with severe diarrhea in 1 case, and
Table 3. Pathologic characteristics

\begin{tabular}{|c|c|c|c|}
\hline & $\operatorname{LAP}(n=35)$ & OS $(n=55)$ & P-value \\
\hline Tumor size $(\mathrm{cm})^{\mathrm{a}}$ & $4.4(3.1)$ & $5.7(2.5)$ & 0.017 \\
\hline Proximal margin $(\mathrm{cm})^{\mathrm{a}}$ & $10.0(3.5)$ & $12.7(7.2)$ & 0.061 \\
\hline Distal margin $(\mathrm{cm})^{\mathrm{a}}$ & $12.5(8.3)$ & $13.7(11.7)$ & 0.617 \\
\hline Retrieved LN (\%) & $13.8(8.8)$ & $15.9(10.7)$ & 0.279 \\
\hline $\begin{array}{l}\text { Histologic differentiation (\%) } \\
\text { Well } \\
\text { Moderate } \\
\text { Poorly }\end{array}$ & $\begin{array}{r}8(22.9) \\
22(62.9) \\
5(14.3)\end{array}$ & $\begin{array}{r}3(5.5) \\
46(83.6) \\
6(10.9)\end{array}$ & 0.035 \\
\hline $\begin{array}{l}\text { Stage }(\%) \\
0 / 1 \\
\| \\
\|\|\end{array}$ & $\begin{array}{r}12(34.3) \\
16(45.7) \\
7(20.0)\end{array}$ & $\begin{array}{c}5(9.1) \\
29(52.7) \\
21(38.2)\end{array}$ & 0.008 \\
\hline
\end{tabular}

LAP, laparoscopic surgery; OS, open left hemicolectomy; LN, lymph node.

a Values are presented as means (standard deviations).

pneumonia due to atelectasis in 2 cases. The total incidences and the major complication rates, classified according to Dindo's classification method, for the two groups were not significantly different.

\section{The number of dissected lymph nodes, the length of the resection margin and recurrence}

The pathological results for the patients of the two groups that underwent surgery for tumors were compared. The mean size of lesions for the LAP group was $4.4 \pm 3.1 \mathrm{~cm}$, and that of the OS group was $5.7 \pm 2.5 \mathrm{~cm}$. The number of dissected lymph nodes for the LAP group was an average of $13.8 \pm 8.8$, and it was less than $15.9 \pm 10.7$ lymph nodes for the OS group, but this difference was not statistically significant $(P=0.279)$. The distance to the proximal resection margin for the LAP group was $10.0 \pm 3.5 \mathrm{~cm}$, and the distance to the distal resection margin was $12.5 \pm 8.3 \mathrm{~cm}$, which were not significantly different from the results for the OS group. Regarding the TNM stages for the LAP group, stage 0 or 1 was observed in 12 cases $(34.3 \%)$, stage 2 in 16 cases (45.7\%), and stage 3 in 7 cases $(20.0 \%)$. In the OS group, stage 0 or 1 was observed in 5 cases (9.1\%), stage 2 in 29 cases (52.7\%), and stage 3 in 21 cases (38.2\%) (Table 3). No study subject patient was lost during the follow-up observation period; the median follow-up observation period of 35 patients of the LAP group was 21 months (range, 1 to 60 months). During that period, none of patients showed local recurrence or recurrence in the trocar site, and 3 patients developed distant metastasis $(8.5 \%)$. In the 3 patients showing systemic recurrence, the liver and the peritoneum were the sites in 1 case, the lung was the site in 1 case and the peritoneum was the site in 1 case. The median interval from surgery to recurrence for the LAP group was 10.3 months (range, 4 to 22 months). On the other hand, the median follow-up observation period for the OS group was 26 months (range, 2 to 60 months), and re- 
currence was observed in 8 patients (14.5\%). In the OS group, anastomosis site recurrence was observed in 1 case, and systemic recurrence was observed in 7 cases. For the 7 patients showing systemic recurrence, the sites of the recurrences were the liver in 2 cases, the liver and the peritoneum in 1 case, the lung in 3 cases, and the iliac lymph node in 1 case.

\section{DISCUSSION}

In our study, the early postoperative outcomes and the usefulness of a laparoscopic left hemicolectomy were analyzed by comparing descending colon cancer patients treated by laparoscopic surgery with those treated with open abdomen surgery. The authors were able to confirm that, in comparison with traditional open abdominal surgery, a laparoscopic left hemicolectomy has clinical advantages in indices such as operation time, transfusion volume during surgery, recovery of bowel movement, and mean hospitalization days and simultaneously that it is safe oncologically regarding for pathological indices of specimens or postoperative complications. Such satisfactory results could be obtained due to an improvement the of surgeon's techniques, systemized surgical methods, and the development of laparoscopic surgical equipment, such as ultrasonic instruments like the Harmonic scalpe ${ }^{\circledR}$ (Ultracision Inc., Smithfield, RI, USA).

The characteristics of the two groups, such as the gender of the two groups, age, body mass index, associated diseases, and locations of tumors were not significantly different; nonetheless, preoperative CEA and TNM stage were different. This is interpreted as being due to our study being a single-institutional retrospective comparisonal study; thus, limitations, such as in the selection of patients, are expected. However, reports on laparoscopic surgery for descending colon cancer are not abundant, and in a multicenter randomized controlled prospective study conducted to assess the safety of laparoscopic surgery for colon cancer, similarly, descending colon cancers, including transverse colon cancers, were excluded $[1,4,9]$. To prove that the surgical outcomes and the oncological results of laparoscopic surgery are not inferior to those for the previous standard open surgery, study methods with the highest reliability, such as prospective randomized controlled trials, are necessary. Nonetheless, since the incidence of descending colon cancer is as low as $5-6 \%$ of all colon cancers, for a comparison and analysis of surgical outcomes, including long-term oncological outcomes, such research designs may be difficult to prospectively apply at a single institution. This study is the largest-scale comparisonal study of laparoscopic left hemicolectomies performed on the descending colon to data until now, and the clinical efficacy of laparoscopic surgery confirmed in our study should be validated in large-scale multicenter prospective studies in the future.

In result of recent studies, resection for descending colon cancer was associated with comparatively longer operation time, longer hospitalization period and higher rates of complications as compared to other procedures; anterior resection and right hemicolectomy. This reflects that a left hemicolectomy including the radical resection of the splenic flexure is technically difficult [10-11]. The authors favored a medial approach that sufficiently resected the descending colon from the retroperitoneum and subsequently exposed the lesser sac by separating the origin of the transverse mesocolon from the pancreas, and in comparison with the traditional lateral approach, such a surgical method is thought to be useful for a safer approach to the lesser sac between the transverse colon and the greater omentum without injuring the spleen.

Surgical techniques for the severance and ligation of the inferior mesenteric vein differ with the investigator. Some investigators expressed the concern that at the time of a left hemicolectomy, ligation of the inferior mesenteric vein might impair venous blood flow. However, the authors did not make any effort to preserve the inferior mesenteric vein specifically because the right drainage route to the internal iliac vein was sufficient and severing the inferior mesenteric vein was advantageous for securing the space and facilitating the surgery. In our study, in most patients, no special complications associated with the impairment of blood flow were experienced during the follow-up observation period; similarly, in abdominal computed tomography performed regularly during the followup observation period, no imaging findings of congested venous blood flow were detected.

The overall complications of the two groups were not statistically different. In our study, regarding the classification of complications, the level of postoperative complications was classified by considering grade I and II described in Table 2 as minor complications, and grade III, IV and V as major complications [12]. In the LAP group, 5 patients developed complications. Among them, in one patient, herniorrhaphy was performed for an incisional hernia 5 months after surgery. In the OS group, 11 patients developed complications. Among them, 1 patient experienced sepsis and an intraperitoneal abscess due to anastomosis leakage. Excluding these two patients, in the two groups, no major complications (under stage 3 of the Dindo's classification) were detected.

In the past, it has been proposed that for transverse colon cancer located in the vicinity of the splenic flexure or for proximal descending colon cancer cases, a more extended resection is required. However, recently, the major opinion is that a radial resection could be anticipated only for a left hemicolectomy. At our hospital, similarly, surgical ranges are determined based on the location of the tumor and the progression level [13-15]. We experienced a total of 35 cases of laparoscopic surgery for descending colon cancer. Among them, in 29 cases (83\%), a standard left hemicolectomy was performed, including a lymphadenectomy with ligation of the left branch of the middle colic 
artery and the left colic artery. In the remaining 6 cases (17\%), surgeries performing high ligation of the inferior mesenteric artery in the origin area and a resection from the proximal descending colon to the sigmoid colon were executed. In our study, to examine the results of a left hemicolectomy for descending colon cancer in more even patient groups, cases involving a segmental resection of transverse colon and cases of colon cancer located in the sigmoid descending colon junction were excluded from the analysis.

In our cases, the number of lymph nodes resected during laparoscopic surgery for descending colon cancer was 13.8 nodes, the length of the proximal resection margin was an average of $10.0 \mathrm{~cm}$, the distal area was $12.5 \mathrm{~cm}$, and sufficient resection margins and lymphadenectomy were shown [16-19]. No case of recurrence in the trocar site or pertinent to the mini-laparotomy site were experienced [20-22]. During the study period, in the LAP group, 3 patients developed systemic recurrence; 1 case of liver metastasis, 1 case of lung metastasis, and 1 case of liver metastasis and multicentric peritoneal metastasis simultaneously. Among them, in 2 patients, distant metastasis was detected within 6 months, and those two patients were TNM stage IIIC patients; thus, rather than cancer that progressed rapidly after surgery, the cancer might have been a micrometastasis that was present prior to surgery and detected later. In this study, we did not compare the survivals of the LAP group and the OS group because the follow-up observation period was relatively short, and the TNM stages of the two groups were somewhat different. However, according to many previous studies, the overall recurrence rate was $8.5 \%$ during the 21-month follow-up period, which allows us to conclude that, from the aspect of survival rate, laparoscopic surgery for descending colon cancer is comparable to open conventional surgery. However, our study only presents the early results for a laparoscopic resection for descending colon cancer, and more cases and long-term follow-up observation are needed.

This study presents the results of a short-term follow-up observation of patients who underwent a laparoscopic left hemicolectomy. It is true that the study has many limitations because it is a single-institution retrospective study; nonetheless, satisfactory results were obtained in regard to operation time, hemorrhage volume, and postoperative clinical outcomes. In spite of that, the radical resection and the oncological safety of the technique should be proven by long-term follow-up observation of chronic complications or recurrence rates, and larger scale multicenter prospective studies of laparoscopic left hemicolectomies is thought to be required. If a laparoscopic left hemicolectomy is performed after a sufficient learning curve for colon cancer in other areas and if it is performed on appropriate patient groups under accurate preoperative diagnosis, it should be a safe and useful treatment for descending colon cancer.

\section{CONFLICT OF INTEREST}

No potential conflict of interest relevant to this article was reported.

\section{REFERENCES}

1. Leung KL, Kwok SP, Lam SC, Lee JF, Yiu RY, Ng SS, et al. Laparoscopic resection of rectosigmoid carcinoma: prospective randomised trial. Lancet 2004;363:1187-92.

2. Guillou PJ, Quirke P, Thorpe H, Walker J, Jayne DG, Smith AM, et al. Short-term endpoints of conventional versus laparoscopicassisted surgery in patients with colorectal cancer (MRC CLASICC trial): multicentre, randomised controlled trial. Lancet 2005; 365:1718-26.

3. Lacy AM, Garcia-Valdecasas JC, Delgado S, Castells A, Taura P, Pique JM, et al. Laparoscopy-assisted colectomy versus open colectomy for treatment of non-metastatic colon cancer: a randomised trial. Lancet 2002;359:2224-9.

4. Clinical Outcomes of Surgical Therapy Study Group. A comparison of laparoscopically assisted and open colectomy for colon cancer. N Engl J Med 2004;350:2050-9.

5. Fleshman J, Sargent DJ, Green E, Anvari M, Stryker SJ, Beart RW $\mathrm{Jr}$, et al. Laparoscopic colectomy for cancer is not inferior to open surgery based on 5-year data from the COST Study Group trial. Ann Surg 2007;246:655-62.

6. Jass JR. Subsite distribution and incidence of colorectal cancer in New Zealand, 1974-1983. Dis Colon Rectum 1991;34:56-9.

7. Toyoda Y, Nakayama T, Ito Y, Ioka A, Tsukuma H. Trends in colorectal cancer incidence by subsite in Osaka, Japan. Jpn J Clin Oncol 2009;39:189-91.

8. Sjo OH, Lunde OC, Nygaard K, Sandvik L, Nesbakken A. Tumour location is a prognostic factor for survival in colonic cancer patients. Colorectal Dis 2008;10:33-40.

9. Lacy AM, Delgado S, Castells A, Prins HA, Arroyo V, Ibarzabal A, et al. The long-term results of a randomized clinical trial of laparoscopy-assisted versus open surgery for colon cancer. Ann Surg 2008;248:1-7.

10. Jamali FR, Soweid AM, Dimassi H, Bailey C, Leroy J, Marescaux J. Evaluating the degree of difficulty of laparoscopic colorectal surgery. Arch Surg 2008;143:762-7.

11. Kuwabara K, Matsuda S, Fushimi K, Ishikawa KB, Horiguchi H, Fujimori K. Quantitative comparison of the difficulty of performing laparoscopic colectomy at different tumor locations. World J Surg 2010;34:133-9.

12. Dindo D, Demartines N, Clavien PA. Classification of surgical complications: a new proposal with evaluation in a cohort of 6336 patients and results of a survey. Ann Surg 2004;240:205-13.

13. Nakagoe T, Sawai T, Tsuji T, Jibiki M, Ohbatake M, Nanashima A, et al. Surgical treatment and subsequent outcome of patients with carcinoma of the splenic flexure. Surg Today 2001;31:204-9.

14. Nakagoe T, Sawa T, Tsuji T, Jibiki M, Nanashima A, Yamaguchi H, 
et al. Carcinoma of the splenic flexure: multivariate analysis of predictive factors for clinicopathological characteristics and outcome after surgery. J Gastroenterol 2000;35:528-35.

15. Sadler GP, Gupta R, Foster ME. Carcinoma of the splenic flexure: a case for extended right hemicolectomy? Postgrad Med J 1992; 68:487.

16. Hong D, Tabet J, Anvari M. Laparoscopic vs. open resection for colorectal adenocarcinoma. Dis Colon Rectum 2001;44:10-8.

17. Braga M, Vignali A, Gianotti L, Zuliani W, Radaelli G, Gruarin P, et al. Laparoscopic versus open colorectal surgery: a randomized trial on short-term outcome. Ann Surg 2002;236:759-66.

18. Milsom JW, Bohm B, Hammerhofer KA, Fazio V, Steiger E, Elson P. A prospective, randomized trial comparing laparoscopic versus conventional techniques in colorectal cancer surgery: a pre- liminary report. J Am Coll Surg 1998;187:46-54.

19. Barlehner E, Benhidjeb T, Anders S, Schicke B. Laparoscopic resection for rectal cancer: outcomes in 194 patients and review of the literature. Surg Endosc 2005;19:757-66.

20. Reilly WT, Nelson H, Schroeder G, Wieand HS, Bolton J, O'Connell MJ. Wound recurrence following conventional treatment of colorectal cancer: a rare but perhaps underestimated problem. Dis Colon Rectum 1996;39:200-7.

21. Silecchia G, Perrotta N, Giraudo G, Salval M, Parini U, Feliciotti F, et al. Abdominal wall recurrences after colorectal resection for cancer: results of the Italian registry of laparoscopic colorectal surgery. Dis Colon Rectum 2002;45:1172-7.

22. Tomita H, Marcello PW, Milsom JW. Laparoscopic surgery of the colon and rectum. World J Surg 1999;23:397-405. 